\title{
ESTIMATIVA DE TAMANHO EFETIVO DE ENDOGAMIA POR MARCADORES GENÉTICOS ${ }^{1}$
}

\author{
Alexandre Magno Sebbenn ${ }^{2}$ e Carlos Eduardo Sicole Seoane ${ }^{3}$
}

\begin{abstract}
RESUMO - Atividades de conservação e melhoramento genético requerem medidas de tamanho efetivo para manutenção e controle do potencial evolutivo das populações sob manipulação. Populações naturais são, muitas vezes, estruturadas e, conseqüentemente, podem apresentar algum grau de endogamia e parentesco. O objetivo deste trabalho foi descrever um simples estimador de tamanho efetivo de endogamia para populações de espécies monóicas com qualquer nível de endogamia e parentesco. O estimador foi derivado, assumindo-se que a endogamia é a mesma em todos os indivíduos da população. Ele pode ser utilizado em populações com pedigree conhecido ou o parentesco médio entre pares de indivíduos da população-alvo pode ser estimado a partir de dados de marcadores genéticos co-dominantes. Um exemplo é dado onde o estimador é aplicado em uma população de uma espécie arbórea monóica, Euterpe edulis.
\end{abstract}

Palavras-chave: Espécies arbóreas, coancestria, parentesco e genética de populações.

\section{ESTIMATIVE OF INBREEDING EFFECTIVE SIZE BY GENETIC MARKERS}

\begin{abstract}
Conservation and genetic improvement activities require measures of effective size in order to maintain and control the evolutive potential of the populations under manipulation. Natural populations are sometimes structured and thus levels of endogamy and relatedness can exist. The aim of this work is to describe an estimator for the inbreeding effective population size of monoecious species populations with any level of endogamy and relatedness. The estimator was derived assuming that endogamy is the same in all individuals of the population. This can be used in populations with known pedigree or the average of the relatedness between pairwise individuals of target populations can be estimated by codominant genetic markers. An example is given in which the estimator is applied to a natural population of a monoecious tree species, Euterpe edulis.
\end{abstract}

Key words: Tree species, coancestry, relatedness and population genetic.

\section{INTRODUÇÃO}

O conceito de tamanho efetivo $\left(N_{e}\right)$ tem papel importante nas teorias evolutiva (WRIGHT, 1938), genética de populações, quantitativa (CABALLERO e HILL, 1992) e conservação genética (SUGG e CHESSER, 1994). O tamanho efetivo teórico pode, contudo, diferir muito do tamanho senso de uma população, sendo, usualmente, muito menor (WRIGHT, 1938). Populações de tamanho finito são objetos de dois efeitos relacionados: primeiro, o efeito da endogamia, que é uma conseqüência da autofecundação ou do cruzamento entre parentes. Em uma população finita, dois alelos homólogos em um indivíduo têm certa probabilidade de descenderem de um mesmo gene de um antecessor recente. Isso leva a uma correlação no conteúdo gênico dos gametas unidos (zigotos) e ao aumento na homozigose

\footnotetext{
${ }^{1}$ Recebido em 07.11.2003 e aceito para publicação em 25.11.2004.

${ }^{2}$ Instituto Florestal de São Paulo, C. Postal 1322, 01059-970, São Paulo, SP. E-mail: <amsebbenn@ bol.com.br>.

${ }^{3}$ ESALQ/USP, AV. Pádua Dias, 11, 13400-970, Piracicaba, SP.
} 
média da população; segundo, o efeito da deriva aleatória nas frequiências alélicas que ocorre devido à variância amostral no processo de transmissão dos alelos de geração para geração, levando ao aumento na homozigose dentro da população e, eventualmente, à extinção e fixação aleatória de alelos (KIMURA e CROW, 1963).

O tamanho efetivo é definido como o tamanho de uma população idealizada que poderia gerar a mesma quantidade de endogamia ou variância nas freqüências alélicas como a observada na população sob consideração (KIMURA e CROW, 1963; CROW e KIMURA, 1970; CABALLERO, 1994). A definição de tamanho efetivo em função da endogamia dá origem ao "tamanho efetivo de endogamia", e a definição em função da variância nas frequiências alélicas dá origem ao "tamanho efetivo de variância". O tamanho efetivo de endogamia trata da população parental (ou avós), enquanto o tamanho efetivo de variância é diretamente relacionado às populações de progênies (KIMURA e CROW, 1963). No presente trabalho, o enfoque será o tamanho efetivo de endogamia.

Estimadores de tamanhos efetivos de endogamia têm sido derivados nas mais diversas situações (WRIGHT, 1938; KIMURA e CROW, 1963; CROW e KIMURA, 1970; LI, 1976, POLLAK, 1987; CROW e DENNISTON, 1988; CABALLERO, 1994; NUNNEY, 1999; VENCOVSKY e CROSSA, 1999, entre outros). O tamanho efetivo é sempre definido em relação a uma população de referência ideal. A população ideal consiste de uma populaçãobase de tamanho infinito, subdividida em infinitas subpopulações de cruzamentos aleatórios, cada uma com número $(N)$ constante de indivíduos reprodutivos por geração. Em cada subpopulação, os indivíduos produzem um infinito número de gametas masculinos e femininos dentro de um grande conjunto, do qual apenas $2 N$ gametas são amostrados e unidos para produzir os $N$ zigotos da seguinte geração. Todos os indivíduos sobrevivem do nascimento à fase adulta. Ambas as amostras dos gametas e sua união (incluindo autofecundação) são aleatórias, com a ressalva de que todos os indivíduos têm iguais chances de produzir progênies, e a distribuição do número de progênies é multinomial. Mudanças sistemáticas nas frequiências alélicas (seleção) são excluídas nessa população idealizada, as gerações não se sobrepõem e apenas locos autossomais são considerados (CABALLERO, 1994).

R. Árvore, Viçosa-MG, v.29, n.1, p.1-7, 2005
Coancestria, parentesco e endogamia são conceitos intimamente relacionados. A coancestria $\left(\theta_{x y}\right)$ é a probabilidade de que dois alelos homólogos, retirados aleatoriamente de dois indivíduos, $x$ e $y$, sejam idênticos por descendência, isto é, sejam cópias de um mesmo alelo de um antecessor recente (LYNCH e WALSH, 1998). Em populações de cruzamentos aleatórios, a coancestria equivale à endogamia na descendência $\left(\theta_{x y}=F\right)$. Essa definição de coancestria inclui o caso em que os dois indivíduos são idênticos, quando se obtém a autocoancestria $\left(\theta_{x x}\right)$, que equivale à endogamia na descendência após autofecundações. Assim, a coancestria média de uma população $(\bar{\theta})$ inclui a autocoancestria e a coancestria entre todos os pares de indivíduos da população (LINDGREN et al., 1997). O parentesco $\left(r_{x y}\right)$ mede a proporção de alelos entre dois indivíduos que são idênticos por descendência. Por exemplo, o parentesco entre o indivíduo $x$ e $y$ é a proporção de alelos em $y$ que são idênticos por descendência aos alelos presentes em $x$; equivalentemente, esta é a probabilidade de que um alelo aleatório amostrado em $y$ seja idêntico por descendência a um alelo presente em $x$. Em espécies diplóides, $r_{x y}=r_{y x}$ e, assim, um alelo aleatório de $y$ tem duas chances de ser idêntico a um alelo em $x$ : este pode ser idêntico a um alelo homólogo de $x$, ou ao outro. Se $x$ não é endogâmico, essas duas probabilidades são independentes e mutuamente exclusivas, e, uma vez que cada homólogo tem a probabilidade $\theta_{x y}$ de ser idêntico por descendência, o parentesco é duas vezes essa probabilidade, $r_{x y}=2 \theta_{x y}(\mathrm{SMITH}, 1999)$. A endogamia $(F)$ é a probabilidade de retirar aleatoriamente dois alelos, em um loco, em um indivíduo $x$ e de eles serem idênticos por descendência (LYNCH e WALSH, 1998). A probabilidade de os alelos em $x$ serem idênticos por descendência depende de autocoancestria da mãe, se $x$ é filho de autofecundação, ou da coancestria entre seus parentais (materno e paterno), se $x$ é filho de cruzamento.

O interesse aqui é a estimativa do tamanho efetivo de endogamia em populações naturais de espécies arbóreas, com genealogia desconhecida, com o intuito de definir tamanhos de populações para conservação genética in situ. Espécies arbóreas muitas vezes apresentam populações estruturadas, devido à dispersão de sementes próximas ou nas vizinhanças da árvorematriz. Como conseqüência, pode haver endogamia e parentesco nas populações. Em uma população de 
$n$ indivíduos, endogamia $\hat{F}$ e sem parentesco, o tamanho efetivo de endogamia pode ser estimado por:

$$
\hat{N}_{e}=\frac{n}{1+\hat{F}} \operatorname{Li}(1976, \text { pág. 562). }
$$

Embora esse estimador considere a endogamia na população, ele desconsidera a presença de parentesco entre os membros da população, que é outro fator gerador de endogamia na descendência. Estimadores que consideram tanto a endogamia quanto o parentesco nas populações são, portanto, desejáveis. Isso é, justamente, o objetivo do presente trabalho, ou seja, descrever um estimador para o tamanho efetivo de endogamia, em populações de espécies monóicas com qualquer grau de endogamia e parentesco intrapopulacional.

\section{MÉTODO}

O tamanho efetivo de endogamia $\left(N_{e}\right)$ foi derivado para indivíduos diplóides, de espécies monóicas, com qualquer grau de endogamia e parentesco dentro das populações, usando o mesmo princípio utilizado por Lindgren et al. (1997) para obter o número status $\left(N_{s}\right)$. Um exemplo numérico do estimador derivado é dado com base em uma amostra de 25 árvores adultas de uma população de Euterpe edulis Martius, localizada em um fragmento denominado Serra do São João, Estado do Rio de Janeiro. O genótipo dos indivíduos foi avaliado a partir de cinco locos microssatélites polimórficos (Seoane, em preparação). Com base nesses dados, para calcular o tamanho efetivo de endogamia, foi estimado o coeficiente de parentesco médio entre todos os pares de indivíduos da população, usando o método de Lynch e Ritland (1999) e o programa Sancho (COELHO, 2001).

\section{RESULTADOS E DISCUSSÃO}

\subsection{Tamanho efetivo de endogamia}

O tamanho efetivo de endogamia $\left(N_{e}\right)$ foi derivado do mesmo princípio utilizado por Lindgren et al. (1997) para obter o número status $\left(N_{s}\right)$. Este é uma medida populacional de representatividade genética semelhante ao tamanho efetivo, contudo diferindo desse em relação à população de referência, a qual corresponde a uma população de genótipos diplóides, de cruzamentos aleatórios, não aparentados e endogâmicos (LINDGREN et al., 1997), no caso do número status; e a população ideal, no caso do tamanho efetivo.
Considere uma população ideal de $N$ indivíduos diplóides, monóicos, reprodutivos e de cruzamentos aleatórios. Nessa população, segundo Crow e Kimura (1970), a variância amostral na frequiência de um alelo $p\left(\hat{\sigma}_{p}^{2}\right)$ é: $\sigma_{p}^{2}=\frac{p(1-p)}{2 N}$

Como na população ideal não existem endogamia e parentesco, o tamanho senso é igual ao tamanho efetivo $\left(N=N_{e}\right)$, e pode-se escrever $\sigma^{2}$ em função de $N_{e}$, de forma que: $\sigma_{p}^{2}=\frac{p(1-p)}{2 N_{e}}$

Para populações com parentesco, contudo, a expressão 3 não é válida. Cockerham (1969) definiu variância amostral na freqüência de um alelo $p\left(\hat{\sigma}_{p}^{2}\right)$ em populações com parentesco, em termos de coancestria $(\theta)$, como: $\sigma_{p}^{2}=\theta p(1-p)$

Igualando as expressões 4 e 3 , obtém-se a estimativa do tamanho efetivo de endogamia em função do coeficiente médio de coancestria da população:

$$
\begin{aligned}
& \theta p(1-p)=\frac{p(1-p)}{2 N_{e}} \\
& N_{e}=\frac{0,5}{\theta}
\end{aligned}
$$

A expressão 5 confunde-se com a definição de número status (LINDGREN et al., 1997) e será a base para a definição do tamanho efetivo de endogamia. O símbolo $\theta$ na expressão 5 será substituído por $\bar{\theta}$ para ressaltar que se trata de um valor médio populacional.

Como comentado, o coeficiente médio de coancestria de uma população $(\bar{\theta})$, em espécies monóicas, depende da autocoancestria $\left(\theta_{x x}\right)$ dos indivíduos, da coancestria $\left(\theta_{x y}\right)$ entre todos os pares de indivíduos e da endogamia dos indivíduos $(F)$. Considere a matriz de coancestria entre $n$ indivíduos de uma população (Quadro 1). A diagonal de tamanho $n$ corresponde à autocoancestria $0,5(1+F)$ ou, em outros termos, à endogamia que poderia ser gerada na descendência pelo cruzamento dos indivíduos consigo mesmos, que é o que se conhece por autofecundação. As partes superior e inferior da diagonal da matriz são simétricas e idênticas, correspondendo à coancestria entre todos os pares de indivíduos da população $\theta_{x y}(1+F)$. O coeficiente médio de coancestria da população $(\bar{\theta})$ corresponde à média de todas as coancestrias da matriz, inclusive dos indivíduos consigo mesmos (autocoancestria):

$$
\bar{\theta}=\frac{n \theta_{x x}(1+F)+\sum_{x=1}^{n} \sum_{y \neq 1}^{n} \theta_{x y}(1+F)}{n^{2}}
$$

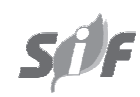

R. Árvore, Viçosa-MG, v.29, n.1, p.1-7, 2005 
Quadro 1 - Matriz de coancestria para $n$ indivíduos de uma simples população Table 1 - Coancestry matrix for $n$ individuals of a simple population

\begin{tabular}{cccccc}
\hline $\mathrm{N}^{0}$ individuos & 1 & 2 & 3 & $\ldots$ & $n$ \\
\hline 1 & $0,5(1+\hat{F})$ & $\hat{\theta}_{12}(1+\hat{F})$ & $\hat{\theta}_{13}(1+\hat{F})$ & $\cdots$ & $\hat{\theta}_{1 n}(1+\hat{F})$ \\
2 & $\hat{\theta}_{12}(1+\hat{F})$ & $0,5(1+\hat{F})$ & $\hat{\theta}_{23}(1+\hat{F})$ & $\ldots$ & $\hat{\theta}_{2 n}(1+\hat{F})$ \\
3 & $\hat{\theta}_{13}(1+\hat{F})$ & $\hat{\theta}_{23}(1+\hat{F})$ & $0,5(1+\hat{F})$ & $\ldots$ & $\hat{\theta}_{3 n}(1+\hat{F})$ \\
$\cdot$ & $\cdot$ & $\cdot$ & $\cdot$ &. &. \\
$\cdot$ & $\cdot$ & $\cdot$ & $\cdot$ &. &. \\
$n$ & $\hat{\theta}_{i 1}(1+\hat{F})$ & $\hat{\theta}_{i 2}(1+\hat{F})$ & $\hat{\theta}_{i 3}(1+\hat{F})$ & $\cdots$ & $0,5(1+\hat{F})$ \\
\hline
\end{tabular}

O termo $n \theta_{x x}(1+F)$ corresponde à autocoancestria (diagonal da matriz do Quadro 1), que é sempre igual a $0,5(1+F)$, e o termo $\sum_{x=1}^{n} \sum_{y \neq 1}^{n} \theta_{x y}(1+F)$ corresponde à soma de todas as coancestrias entre os pares de indivíduos da matriz (Quadro 1). Substituindo a expressão 6 na 5, obtém-se um estimador de tamanho efetivo em populações com qualquer nível de endo-

$$
\text { gamia e coancestria: } N_{e}=\frac{0,5 n^{2}}{(1+F)\left(0,5 n+\sum_{x=1}^{n} \sum_{y \neq 1}^{n} \theta_{x y}\right)}
$$

Por exemplo, considere uma "população" com 10 indivíduos não-endogâmicos e não-aparentados entre si $\left(F=\theta_{x y}=0\right)$. Essa população têm 10 autocoancestrias iguais a 0,5 e 90 pares de coancestrias [ $n(n-1)]$ iguais a zero. Substituindo esses valores na expressão 7, tem-se que o tamanho efetivo é igual a 10, ou seja, os 10 indivíduos da presente população representam 10 indivíduos de uma população ideal. Considerando, agora, $10 \%$ de endogamia média na população, o tamanho efetivo será de 9,09, ou seja, os 10 indivíduos representam nove indivíduos de uma população ideal. O mesmo resultado poderia ser obtido utilizando-se a expressão 1 , visto que na população exemplificada não existe parentesco, e o tamanho efetivo é, nesse caso, função apenas do coeficiente de endogamia. Finalmente, considere que os 10 indivíduos são irmãos de autofecundação, de um parental nãoendogâmico $(F=0)$; portanto, a coancestria entre cada par é igual a 0,5 e o somatório das coancestrias, igual a 45 [0,5x10(10-1)], de forma que o tamanho efetivo é igual a 1, isto é, os 10 irmãos de autofecundação representam apenas um indivíduo de uma população ideal.

R. Árvore, Viçosa-MG, v.29, n.1, p.1-7, 2005

\footnotetext{
R. Árvore, Viçosa-MG, v.29, n.1, p.1-7, 2005
}

\subsection{Estimadores de parentesco e endogamia por marcadores genéticos}

Utilizando marcadores genéticos co-dominantes, o coeficiente de coancestria entre pares de indivíduos de uma população pode ser estimado a partir do coeficiente de parentesco $\left(r_{x y}\right)$ entre os pares de indivíduos, usandose uma das diversas medidas de parentesco descritas na literatura, como Lynch (1988), Queller e Goodnight (1989), Ritland (1996), Lynch e Ritland (1999), Wang (2002) e Milligan (2003). Não é objetivo aqui descrever cada um desses estimadores, mas ressalta-se que cada um tem suas vantagens e desvantagens. Por exemplo, o estimador de Queller e Goodnight (1989) é limitado para locos dialélicos, sendo indefinido no caso em que um indivíduo é heterozigoto (LYNCH e RITLAND, 1999). Os estimadores de Lynch e Ritland (1999) e Wang (2002) assumem populações em equilíbrio de HardyWeinberg. Lynch e Ritland (1999), Casteele et al. (2001) e Wang (2002) compararam e discutiram alguns desses estimadores. O que é fato comum entre todos os métodos é a necessidade de grande número de locos e alelos para a obtenção de estimativas confiáveis (baixos erros associados). Existem também vários programas computacionais para o cálculo desses coeficientes, que são distribuídos livremente na rede de computadores, como o programa REL (RITLAND, 2000) e Sancho (COELHO, 2001), entre outros. Considerando que em populações não-endogâmicas o coeficiente de parentesco estima o dobro do coeficiente de coancestria, $r_{x y}=2 \theta_{x y}$ (LYNCH e RITLAND, 1999); logo este último pode ser calculado por, $\theta_{x y}=r / 2$, e a expressão 7 torna-se:

$$
\hat{N}_{e}=\frac{n^{2}}{(1+\hat{F})\left(n+\sum_{x=1}^{n} \sum_{y \neq 1}^{n} \hat{r}_{x y}\right)}
$$



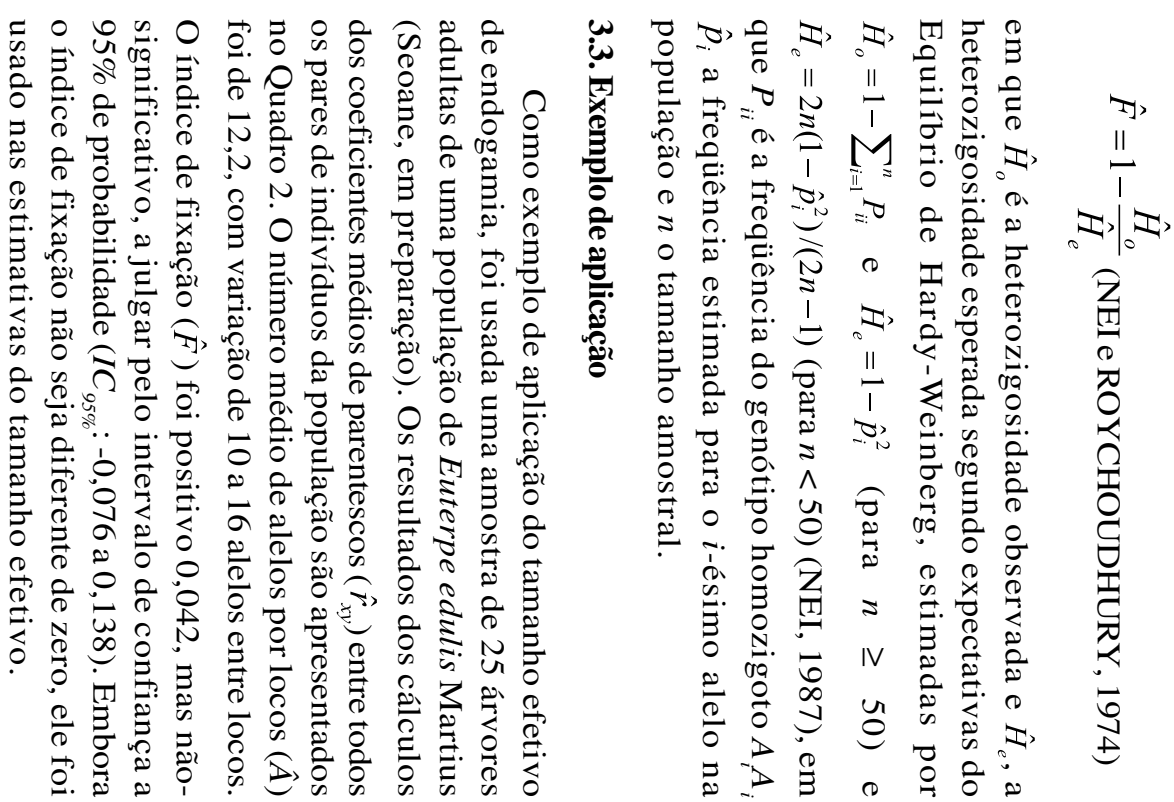

Quadro 2 - Matriz de parentesco $\left(\hat{r}_{x y}\right)$ entre todos os pares de indivíduos de uma população de E. edulis, estimativas obtidas com base em Lynch e Ritland (1999) Table 2 - Matrix of relatedness among all individuals pairwise of a Euterpe edulis population, estimates obtained with base in Lynch e Ritland (1999)


25 


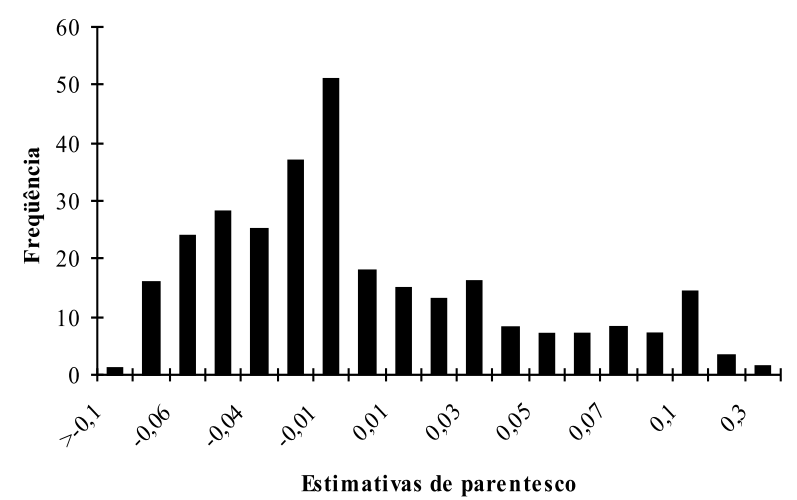

Figura 1 - Distribuição das estimativas de parentesco entre pares de indivíduos de uma população de 25 árvores de E. edulis.

Figure 1 -Distribution of estimates of pairwise relatedness among 25 trees of a population of E. edulis.

Quadro 3-Estimativa de coeficientes de parentesco, coancestria média da população e tamanho efetivo de endogamia

Table 3 - Estimative of the coefficient of relatedness, average coancestry and inbreeding effective size

\begin{tabular}{lccc}
\hline Parâmetros & $\begin{array}{c}\text { Todos } \\
\text { valores }\end{array}$ & $\begin{array}{c}\text { Valores } \\
>0\end{array}$ & $\begin{array}{c}\text { Valores } \\
>0,05\end{array}$ \\
\hline $\begin{array}{l}\text { Tamanho } \\
\text { amostral }-n\end{array}$ & 25 & 25 & 25 \\
$\begin{array}{l}\text { Coeficiente } \\
\text { de parentesco }-\hat{r}_{x y}\end{array}$ & $-0,002$ & 0,005 & 0,003 \\
$\begin{array}{l}\text { Coeficiente médio } \\
\text { de coancestria }-\hat{\theta}\end{array}$ & 0,019 & 0,026 & 0,024 \\
$\begin{array}{l}\text { Tamanho efetivo } \\
\text { de endogamia }-\hat{N}_{e}\end{array}$ & 26,4 & 19,6 & 20,7 \\
\hline
\end{tabular}

Os coeficientes médios de parentesco e de coancestria da população foram baixos em todos os casos. No primeiro caso, o tamanho efetivo de endogamia $(26,4)$ foi superior ao tamanho senso $(25)$, visto que a estimativa média de parentesco na população foi negativa $(-0,002)$. No segundo caso, considerandose somente os valores positivos, o tamanho efetivo foi de 19,6. A interpretação seria de que os 25 indivíduos da referida população correspondem a 20 indivíduos de uma população ideal, ou seja, a 20 indivíduos de uma população de cruzamentos aleatórios infinitamente grande, sem seleção, migração, mutação, parentesco e endogamia.
Como observado, a estimativa média de parentesco poderá ser negativa se as estimativas negativas forem consideradas na análise, o que resultará em tamanho efetivo maior do que o número senso. Assim, sugerese, para a obtenção de valores conservadores, considerar os valores negativos como zero e utilizar somente os valores positivos nos cálculos. Com essa estratégia, logicamente ambas as estimativas médias de parentesco e de coancestria aumentam e o tamanho efetivo será menor do que o número senso, caso existam valores positivos na matriz de parentesco. Se todos os valores forem negativos, assumindo-se esses como zero, o tamanho efetivo será igual ao número senso.

Outra estratégia seria utilizar um valor de corte, ou seja, um valor mínimo de parentesco que fosse considerado como mensurador da relação genética entre os pares de indivíduos. Dessa forma, os resultados seriam intermediários entre considerar todos os valores (inclusive os negativos) e somente os positivos. Por exemplo, considerando-se apenas os valores positivos maiores do que 0,05 (valor de corte), ocorre pequena redução nas respectivas estimativas de parentesco, da coancestria média da população e de aumento do tamanho efetivo (Quadro 3). Levando-se em conta que estimativas conservadoras (subestimadas) de tamanho efetivo são mais desejáveis do que estimativas otimistas (superestimadas), por apresentar um quadro menos favorável em programas de conservação genética, sugere-se que se adote a segunda estratégia, isto é, considerem-se os valores negativos como zero e utilizem somente os valores positivos nos cálculos do tamanho efetivo.

\section{CONCLUSÕES}

É possível estimar o tamanho efetivo de endogamia em populações naturais com qualquer nível de endogamia e parentesco, desde que esses parâmetros sejam conhecidos ou estimados via dados de marcadores genéticos co-dominantes.

\section{REFERÊNCIAS BIBLIOGRÁFICAS}

CABALLERO, A. Developments in the prediction of effective population size. Heredity, v. 73, p. 657-679, 1994.

CABALLERO, A.; HILL, W.G. A note on the inbreeding effective population size. Evolution, v. 46, n. 6, p. 1969-1972, 1992. 
CASTEELE, T.V.; GALBUSERA, P.; MATTHYSEN, E. A comparation of microssatellite-based pairwise relatedness estimators. Molecular Ecology, v. 10, p. 1539-1549, 2001.

COCKERHAM, C.C. Variance of gene frequencies. Evolution, v.23, p.72-84, 1969.

COELHO, A.S.G. SANCHO: Sistema de análise para cálculo de herdabilidade e outros parâmetros (software). Goiania, Universidade Federal de Góias, 2001.

CROW, J.F; KIMURA, M.A. An introduction to population genetics theory. London: Harper Row, 1970. 591 p.

CROW, J.F.; DENNISTON, C. Inbreeding and variance effective population numbers.

Evolution, v. 42, p. 482-495, 1988.

KIMURA, M.; CROW, J.F. The effective population number. Evolution, v.17, n.3, p.279$288,1963$.

LI, C.C. Population genetics.Chigago: University Chicago Press, 1976. 366 p.

LINDGREN, D.; LUIGI, D. G.; JEFFERSON, P. A. Status number for measuring genetic diversity. Forest Genetics, v.4, n.2, p.69-76, 1997.

LYNCH, M. Estimation of relatedness by DNA fingerprinting. Molecular Biology Evolution, v.5, p.584-599, 1988.

LYNCH, M.; WALSH, B. Genetics and analysis of quantitative triats. 1 st. Sinauer Associates, Sundeland, 1998.

LYNCH, M.; RITLAND, K. Estimation of pairwise relatedness with molecular markers. Genetics, v.152, p.1753-1766, 1999.

MILLIGAN, B.G. Maximum-likelihood estimation of relatedness. Genetics, v. 163, p.1153-1167, 2003.
NEI, M. Molecular evolutionary genetics. New York: Columbia University Press, 1987.

NEI, M.; ROYCHOUDHURY, A.K. Sampling variances of heterozigosity and genetic distance. Genetics, v.76, p.927-943, 1974.

NUNNEY, L. The effective size for a hierarchically structured population. Evolution, v.53, n. 1, p. $1-10,1999$.

POLLAK, E. On the theory of partially inbreeding finite populations. I partial selfing. Genetics, v. 117, p. 353-360, 1987.

QUELLER, D.C.; GOODNIGNT, K.F. Estimating relatedness using genetic markers. Evolution, v.43, n.2, p. 258-275, 1989.

RITLAND, K. Estimators for pairwise relatedness and individual inbreeding coefficients. Genetics Resources, v.67, p.175-185, 1996.

RITLAND, K. Marker-inferred relatedness as a tool for detecting heritability in nature.

Molecular Ecology, v. 9, p.1195-1204, 2000.

SMITH, J.M. Evolutionary genetics. Oxford: Oxford University Press, 1999. 328 p.

SUGG, D.W.; CHESSER, R.K. Effective population size with multiple paternity. Genetics, v. 137, p. 1147-1155, 1994.

VENCOVSKY, R.; CROSSA, J. Variance effective population size under mixed self and random mating with applications to genetic conservation of species. Crop Science, v.39, p.282-1294, 1999.

WANG, J. An estimator for pairwise relatedness using molecular markers. Genetics, v. 160, p. 1203-1215, 2002.

WRIGHT, S. Size of population and breeding structure in relation to evolution. Science, v. 87 , n. 2263, p. 430-2264, 1938. 
